\title{
Traumatic Pseudoaneurysm of Left Anterior Descending Artery Caused by Thoracic Stab Wound Treated with Coil
}

\section{Ata Firozi ${ }^{1 *}$, Omid Shafee ${ }^{2}$, Aliasghar Farsavian ${ }^{2}$, Hamidreza Sanati ${ }^{1}$, Farshad Shakerian ${ }^{1}$, Reza Kiani ${ }^{1}$, Ali Zahedmehr ${ }^{1}$ and Mona Heidarali $^{3}$}

${ }^{1}$ Associate Professor of Cardiology, Intervention research center, Rajaie Cardiovascular, Medical and Research Center, Iran university of Medical Sciences, Tehran, Iran ${ }^{2}$ Fellow of intervention, Intervention research center, Rajaie Cardiovascular, Medical and Research Center, Iran university of Medical Sciences, Tehran, Iran ${ }^{3}$ Cardiac Electrophysiology Research Center, Rajaie Cardiovascular, Medical and Research Center, Iran University of medical sciences, Tehran, Iran

\begin{abstract}
Trauma is one of the most common causes of death worldwide. Penetrating cardiac injury (PCl) is highly lethal and represents an increasingly important form of traumatic injury. We describe a 25 year-old man suffered stab wound and $\mathrm{PCl}$, as a result of which he underwent surgical thoracotomy for tamponade. He was discharged in good clinical condition and no cardiac perforation was detected. Two weeks later, he presented to our center with prolonged chest pain. Electrocardiography (ECG) and cardiac biomarkers confirmed myocardial damage. Echocardiography showed hypokinesia and thinning $(0.6 \mathrm{~mm})$ of the inferoapical wall segment and mildly reduced left ventricular systolic function. Angiography revealed patent left main coronary artery, LAD, left circumflex, and right coronary artery, along with an abnormal aneurysmal structure at the distal part of the LAD. Coil embolization of the distal LAD pseudoaneurysm was performed which was placed to occlude the proximal entry site of the pseudoaneurysm. Angiography confirmed the disappearance of the pseudoaneurysm. The patient was discharged after 48 hours. Follow-up angiography after two months showed no residual leakage across the vessel. Coil embolization of a distal coronary pseudoaneurysm can be performed as an alternative procedure instead of surgical ligation of the small vessels, with good results. It should be emphasized that one should occlude both proximal and distal entry sites to avoid blood leakage.
\end{abstract}

Keywords: Penetrating cardiac injury; Traumatic; Pseudoaneurysm; Left anterior descending artery; Angiography; Coil

\section{Introduction}

Trauma is one of the most common causes of death worldwide. Penetrating cardiac injury (PCI) is highly lethal and represents an increasingly important form of traumatic injury. A patient with a traumatic laceration of the coronary artery usually does not live long enough to benefit from either diagnostic investigation or surgical therapy [1]. Patients who survived may consequently have a myocardial infarction or a localized aneurysm of the coronary artery. This report describes a patient who, after a stab wound of the chest, had an aneurysm of the left anterior descending coronary artery. All of these lesions were successfully treated by operation 4 years after the injury. This is believed to be the one of the few cases of a traumatic coronary arteriocameral fistula. A few cases of traumatic aneurysm of the left coronary artery and a traumatic left coronary arterial-left ventricular fistula have been reported so far [1].

\section{Clinical Case}

A 25-year-old man suffered a thoracic stab wound and PCI, as a result of which he underwent surgical thoracotomy for tamponade treatment. During surgery, no cardiac perforation was detected, so he was discharged in good clinical condition.

Two weeks later, he presented to our center with prolonged chest pain. Electrocardiography (ECG) and cardiac biomarkers confirmed myocardial damage (Figure 1); he was, therefore, admitted for further evaluation. Echocardiography showed hypokinesia and thinning $(0.6$ $\mathrm{mm}$ ) of the inferoapical wall segment (a marker of non-viable tissue) and mildly reduced left ventricular systolic function (left ventricular ejection fraction of about 45\%). On transesophageal echocardiography, there was no evidence of left ventricular aneurysm or pseudoaneurysm, and the aortic root and arch were normal. The patient was subjected to diagnostic coronary angiography for further evaluation. Angiography revealed patent left main coronary artery, LAD, left circumflex, and right coronary artery, along with an abnormal aneurysmal structure at the distal part of the LAD near the apex, in favor of the pseudoaneurysm of the LAD (Figure 2). No extravasation of the contrast agent into the pericardium was present.

Accordingly, decision was made to perform coil embolization of the distal LAD pseudoaneurysm. The LAD was wired first, and then an ev3 7-30 coil was placed to occlude the proximal entry site of the pseudoaneurysm. There was a residual flow through the distal portion of the vessel and the pseudoaneurysm sac, necessitating the placement of a second coil to occlude the distal entry site of the pseudoaneurysm (Figures 3 and 4). Thereafter, angiography confirmed the disappearance of the pseudoaneurysm while the distal portion of the LAD was filled retrogradely, with a thrombolysis in Myocardial Infarction (TIMI) flow grade of 1-2 (Figure 5).

The procedure and the hospital course were free of complications and symptoms, and the patient was discharged after 48 hours. Followup angiography after two months showed no residual leakage across the vessel. The Figure 6 depicted the follow-up angiography which showed in this lateral view angiogram, no pseudoaneurysm is evident.

*Corresponding author: Ata Firozi, Intervention research center, Rajaie Cardiovascular, Medical and Research Center, Iran University of medical sciences Tehran, Iran, Tel: 0098021-23923017; E-mail: atafirouzi@yahoo.com

Received June 26, 2014; Accepted August 27, 2014; Published September 03, 2014

Citation: Firozi A, Shafee O, Farsavian A, Sanati H, Shakerian F, et al. (2014) Traumatic Pseudoaneurysm of Left Anterior Descending Artery Caused by Thoracic Stab Wound Treated with Coil. J Cardiovasc Dis Diagn 2: 173. doi:10.4172/23299517.1000173

Copyright: (๑) 2014 Firozi A, et al. This is an open-access article distributed under the terms of the Creative Commons Attribution License, which permits unrestricted use, distribution, and reproduction in any medium, provided the original author and source are credited. 
Citation: Firozi A, Shafee O, Farsavian A, Sanati H, Shakerian F, et al. (2014) Traumatic Pseudoaneurysm of Left Anterior Descending Artery Caused by Thoracic Stab Wound Treated with Coil. J Cardiovasc Dis Diagn 2: 173. doi:10.4172/2329-9517.1000173

Page 2 of 3

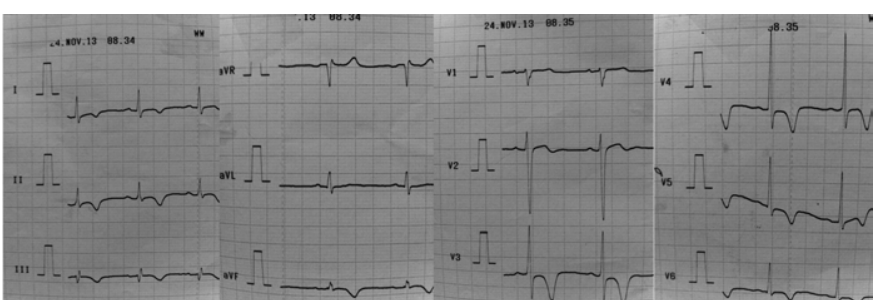

Figure 1: Twelve-lead ECG of the patient, showing evident T inversions of the inferior and pericardial leads.

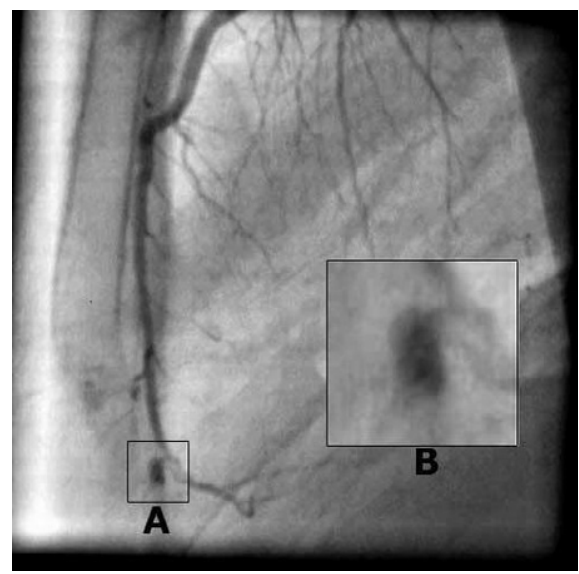

Figure 2: Left lateral projection frozen-frame angiogram of the left system, illustrating a saccular lesion at the distal part of the left anterior descending coronary artery (LAD) (rectangle A). Note that its much narrowed neck is connected to the distal part of the LAD and its body is filled with the contrast agent (rectangle B).

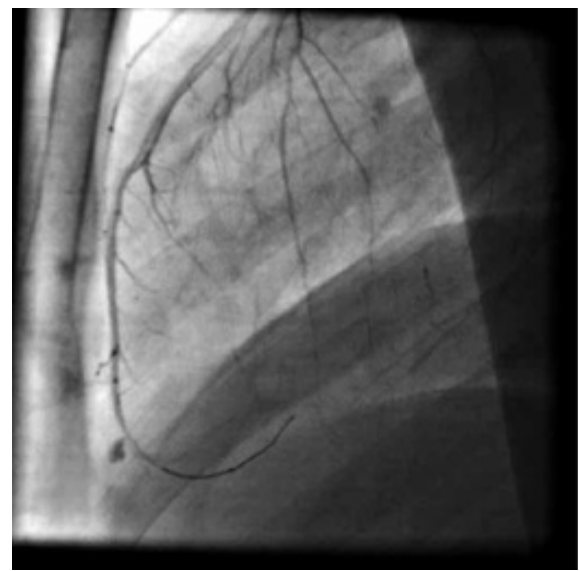

Figure 3: Left anterior descending coronary artery is wired. In this left lateral projection frozen-frame angiogram, the wire is passed distal to the pseudoaneurysm without entering it. Entry to such a place can cause perforation and tamponade.

Distal portion of the left anterior descending coronary artery has a TIMI flow grade of 1-2.

\section{Discussion}

Coronary artery injury due to PCI is not very common $(3.1 \%$ $4.4 \%)$ and mandates surgical or interventional treatment $[2,3]$. PCI is highly lethal and its incidence is on the increase. Massive PCI causes serious damage to the cardiac chambers, with the victim expiring before reaching the trauma bay. Patients with PCI can be classified into 5 groups: lifelessness, critical instability, cardiac tamponade, thoracoabdominal injury, and benign injury $[4,5]$. The most common clinical manifestations of PCI include hemorrhage and pericardial tamponade [6]. Despite the fatal nature of PCI, surprisingly $70 \%$ of the victims do not exhibit symptoms suggestive of cardiac injury [7]. Emergent paraclinical evaluations of patients suspicious of PCI are mandated and include echocardiography, ECG, computed tomography (CT)-scan, and catheterization. In a series of patients with juxtacardiac penetrating chest wounds and stable vital signs, twodimensional echocardiography was found to be $90 \%$ sensitive and $97 \%$ specific for the diagnosis of PCI [7]. Emergent pericardiocentesis is a life-saving measure and is indicated in clinical tamponade. Injuries to the coronary arteries occur in $3.1 \%-4.4 \%$ of PCI cases $[2,3]$. The most frequently involved vessel is the $\mathrm{LAD}$, followed by the right coronary artery $[8,9]$. PCI in the proximity of the coronary arteries should be repaired with pledgeted mattress sutures to prevent occlusion. Injured small peripheral coronary arteries should be ligated, but injured large

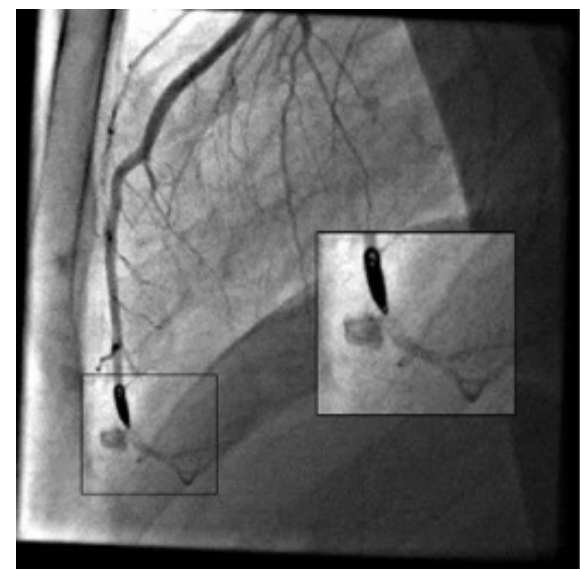

Figure 4: The first ev 3 7-30 coil is placed at the proximal entry site of the pseudoaneurysm, but it is still filled with the contrast agent via the retrograde flow of the distal part of the left anterior descending coronary artery filling.

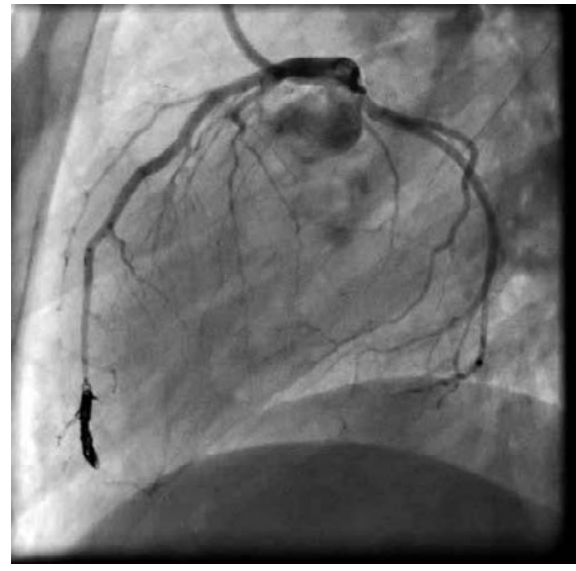

Figure 5: Final angiographic result, after the placement of a second coil (the same coil) to occlude the distal entry site. The pseudoaneurysm disappears with a reduced flow at the distal part (TIMI 1-2), which would be totally obscure in a few moments. 


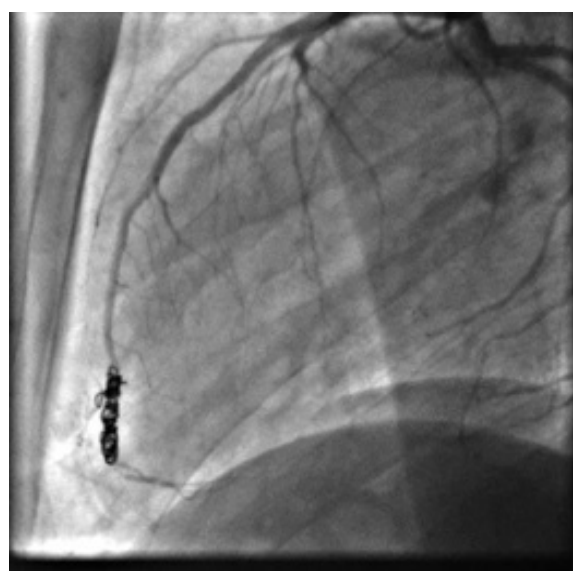

Figure 6: Follow-up angiography. In this lateral view angiogram, no pseudoaneurysm is evident. Distal portion of the left anterior descending coronary artery has a TIMI flow grade of 1-2.

vessels supplying a large portion of the myocardium should be repaired $[10]$.

In our patient, the pseudoaneurysm was prone to life-threatening rupture if left untreated; treatment was, therefore, clearly indicated. There are two treatment options for traumatic coronary artery injuries: surgical repair and intervention. Because the distal part of the LAD (the site of pseudoaneurysm formation) was small and perfused a relatively small territory, the interventional approach seemed feasible with less morbidity than surgical ligation of the LAD or bypass graft surgery. We opted to perform percutaneous occlusion of the pseudoaneurysm. There were two interventional approaches to the treatment of this lesion: coil embolization and stent graft placement. As the distal part of the LAD was small and it seemed that there was not enough viable tissue at the territory, economic considerations favored using coil embolization rather than stent graft implantation. This approach is in contrast with that chosen by Soon Jun Hong et al. for the treatment of a patient with LAD pseudoaneurysm [11]. Citing that injury to the mid part of the LAD had jeopardized a large portion of the myocardium, the authors argued that the aneurysm could not be ligated or embolized with coils. Thus, they used a polytetrafluoroethylene (PTFE) stent graft to seal the lesion and succeeded in achieving a good final result. During the procedure, we utilized a single coil so as to avoid injury to the pseudoaneurysm wall; however, the continuation of the flow through the distal entry site necessitated the use of another coil for occlusion (Figure 3-5). The final angiographic result was excellent, with no residual leakage after the index procedure and also at two months' follow-up. The figure 6 depicted the follow-up angiography showing in this lateral view angiogram, no pseudoaneurysm is evident. Distal portion of the left anterior descending coronary artery has a TIMI flow grade of 1-2.

\section{Conclusion}

Coil embolization of a distal coronary pseudoaneurysm can be performed as an alternative procedure instead of surgical ligation of the small vessels, with good results. It should be emphasized that one should occlude both proximal and distal entry sites to avoid blood leakage.

\section{Acknowledgements}

We would like to thank the cardiac electrophysiology research center for the support of providing this manuscript. This project is support also by the fund of Iran University of medical sciences. We also thank Mrs. Nushin Ashrafi for assisting with providing the additional data for the manuscript.

\section{References}

1. Cheng TO, Adkins PC (1973) Traumatic aneurysm of left anterior descending coronary artery with fistulous opening into left ventricle and left ventricular aneurysm after stab wound of chest. Report of case with successful surgical repair. Am J Cardiol 31: 384-390.

2. Rea WJ, Sugg WL, Wilson LC, Webb WR, Ecker RR (1969) Coronary artery lacerations; an analysis of 22 patients. Ann Thorac Surg 7: 518-528.

3. Demetriades D, van der Veen BW (1983) Penetrating injuries of the heart: experience over two years in South Africa. J Trauma 23: 1034-1041.

4. Saadia R, Levy RD, Degiannis E, Velmahos GC (1994) Penetrating cardiac injuries: clinical classification and management strategy. Br J Surg 81: 1572 1575.

5. Bowley DM, Saeed M, Somwe D, Boffard KD, Naidoo K, et al. (2002) Off-pump cardiac revascularization after a complex stab wound. Ann Thorac Surg 74: 2192-2193.

6. Harman PK, Trinkle JK (1991) Injury to the Heart. In: Trauma, (2nd Edn.) Moore EE, Mattox KL, Feliciano DV (Eds.), Norwalk: Appleton \& Lange.

7. Jimenez E, Martin M, Krukenkamp I, Barrett J (1990) Subxiphoid pericardiotomy versus echocardiography: A prospective evaluation of the diagnosis of occult penetrating cardiac injury. Surgery 108: 676-679.

8. Symbas PN (1991) Cardiothoracic Trauma. Philadelphia: WB Saunders, USA

9. Demetriades D, van der Veen BW (1983) Penetrating injuries of the heart: experience over two years in South Africa. J Trauma 23: 1034-1041.

10. Pons F, Lang-Lazdunski L, de Kerangal X, Chapuis O, Bonnet PM, et al. (2002) The role of videothoracoscopy in management of precordial thoracic penetrating injuries. Eur J Cardiothorac Surg 22: 7-12.

11. Hong SJ, Lim DS, Kim YH, Shim WJ, Park CG, et al. (2005) Chest stab woundrelated coronary artery pseudoaneurysm sealed with a polytetrafluoroethylenecovered stent. Heart Vessels 20: 233-235 